\title{
Lipschitz Spaces and Fractional Integral Operators Associated with Nonhomogeneous Metric Measure Spaces
}

\author{
Jiang Zhou and Dinghuai Wang \\ Department of Mathematics, Xinjiang University, Urumqi 830046, China \\ Correspondence should be addressed to Jiang Zhou; zhoujiangshuxue@126.com
}

Received 1 December 2013; Revised 3 April 2014; Accepted 5 April 2014; Published 17 April 2014

Academic Editor: S. A. Mohiuddine

Copyright (C) 2014 J. Zhou and D. Wang. This is an open access article distributed under the Creative Commons Attribution License, which permits unrestricted use, distribution, and reproduction in any medium, provided the original work is properly cited.

\begin{abstract}
The fractional operator on nonhomogeneous metric measure spaces is introduced, which is a bounded operator from $L^{p}(\mu)$ into the space $L^{q, \infty}(\mu)$. Moreover, the Lipschitz spaces on nonhomogeneous metric measure spaces are also introduced, which contain the classical Lipschitz spaces. The authors establish some equivalent characterizations for the Lipschitz spaces, and some results of the boundedness of fractional operator in Lipschitz spaces are also presented.
\end{abstract}

\section{Introduction}

As we know, the theory on spaces of homogeneous type is needed to assume that measure $\mu$ of metric spaces $(\mathscr{X}, d, \mu)$ satisfies the doubling measure condition, which means that there exists a constant $C$, such that, for every ball $B(x, r)$ of center $x$ and radius $r, \mu(B(x, 2 r)) \leq C \mu(B(x, r))$. In recent years, many classical theories have been proved still valid without the assumption of doubling measure condition; see [1-12]. Recall that a Radon measure $\mu$ on $R^{d}$ is said to only satisfy the polynomial growth condition, if there exists a positive constant $c$ such that, for all $x \in \mathbb{R}^{d}$ and $r>0$, $\mu(B(x, r)) \leq c r^{n}$, where $n$ is some fixed number in $(0, d]$ and $B(x, r)=\left\{y \in \mathbb{R}^{d}:|x-y|<r\right\}$. The analysis associated with such nondoubling measures $\mu$ is proved to play a striking role in solving the long-standing open Painlevés problem by Tolsa [13]. Obviously, the nondoubling measure $\mu$ with the polynomial growth condition may not satisfy the wellknown doubling condition, which is a key assumption in harmonic analysis on spaces of homogeneous type. In 2010, Hytönen [14] introduced a new class of metric measure spaces satisfying both the so-called geometrically doubling and the upper doubling conditions (see the definition below), which are called nonhomogeneous spaces. Recently, many classical results have been proved still valid if the underlying spaces are replaced by the nonhomogeneous spaces of Hytönen (see $[4-6,9-12])$.
Let $(\mathscr{X}, d, \mu)$ be a nonhomogeneous metric measure space in the sense of Hytönen [14]. In this paper, we establish the definition of fractional operator on nonhomogeneous metric measure spaces, which contains the classical fractional integral operator introduced by García-Cuerva and Gatto [7], and similar to the definition introduced by $\mathrm{Fu}$ et al. [11], then we get the $\left(L^{p}(\mu), L^{q, \infty}(\mu)\right)$-boundedness for fractional integral operator on nonhomogeneous metric measure spaces. In Section 3, we also establish the definition of Lipschitz spaces on nonhomogeneous metric measure spaces, which contains the classical Lipschitz spaces. We establish some equivalent characterizations for the Lipschitz spaces. In Section 4, we present some results of the boundedness of fractional operator in Lipschitz spaces.

To state the main results of this paper, we first recall some necessary notions and remarks.

Definition 1 (see [15]). A metric space $(\mathscr{X}, d, \mu)$ is said to be geometrically doubling if there exists some $N_{0} \in \mathbb{N}$ such that, for any ball $B(x, r) \subset \mathscr{X}$, there exist a finite ball covering $\left\{B\left(x_{i}, r / 2\right)\right\}_{i}$ of $B(x, r)$ such that the cardinality of this covering is at most $N_{0}$.

Definition 2 (see [14]). A metric measure space $(X, d, \mu)$ is said to be upper doubling if $\mu$ is a Borel measure on $\mathscr{X}$ and there exist a dominating function $\lambda: \mathscr{X} \times(0, \infty) \rightarrow(0, \infty)$ 
and a positive constant $c_{\lambda}$ such that, for each $x \in \mathscr{X}, r \rightarrow$ $\lambda(x, r)$ is nondecreasing and

$$
\mu(B(x, r)) \leq \lambda(x, r) \leq c_{\lambda} \lambda\left(x, \frac{r}{2}\right) \quad \forall x \in \mathscr{X}, r>0 .
$$

A metric measure space $(\mathscr{X}, d, \mu)$ is called a nonhomogeneous metric measure space if $(\mathscr{X}, d, \mu)$ is geometrically doubling and $(\mathscr{X}, d, \mu)$ is upper doubling.

Remark 3. (i) Obviously, a space of homogeneous type is a special case of upper doubling spaces, where we take the dominating function $\lambda(x, r):=\mu(B(x, r))$. On the other hand, the Euclidean space $\mathbb{R}^{d}$ with any Radon measure $\mu$ as in (1) is also an upper doubling space by taking the dominating function $\lambda(x, r):=C r^{k}$.

(ii) Let $(\mathscr{X}, d, \mu)$ be upper doubling with $\lambda$ being the dominating function on $\mathscr{X} \times(0, \infty)$ as in Definition 2. It was proved in [6] that there exists another dominating function $\tilde{\lambda}$ such that $\widetilde{\lambda} \leq \lambda$ and, for all $x, y \in \mathscr{X}$ with $d(x, y) \leq r$,

$$
\tilde{\lambda}(x, r) \leq C_{\widetilde{\lambda}} \tilde{\lambda}(y, r) .
$$

Thus, in this paper, we always suppose that $\lambda$ satisfies (2).

Definition 4 (see [14]). Let $\alpha, \beta_{\alpha} \in(1, \infty)$. A ball $B \subset X$ is called $(\alpha, \beta)$-doubling if $\mu(\alpha B) \leq \beta_{\alpha} \mu(B)$.

As stated in lemma of [4], there exist plenty of doubling balls with small radii and with large radii. In the rest of the paper, unless $\alpha$ and $\beta_{\alpha}$ are specified otherwise, by an $\left(\alpha, \beta_{\alpha}\right)$ doubling ball we mean a $\left(6, \beta_{6}\right)$-doubling with a fixed number $\beta_{6}>\max \left\{C_{\lambda}^{3 \log _{2} 6}, 6^{n}\right\}$, where $n=\log _{2} N_{0}$ is viewed as a geometric dimension of the spaces.

Definition 5 (see [11]). Let $\epsilon \in(0, \infty)$. A dominating function $\lambda$ is satisfying the $\epsilon$-weak reverse doubling condition if, for all $r \in(0,2 \operatorname{diam}(\mathscr{X}))$ and $a \in(1,2 \operatorname{diam}(\mathscr{X}) / r)$, there exists a number $C(a) \in[1, \infty)$, depending only on $a$ and $\mathscr{X}$, such that, for all $x \in \mathscr{X}$,

$$
\lambda(x, \text { ar }) \geq C(a) \lambda(x, r)
$$

and, moreover,

$$
\sum_{k=1}^{\infty} \frac{1}{\left[C\left(a^{k}\right)\right]^{\epsilon}}<\infty
$$

Remark 6. (i) It is easy to see that if $\epsilon_{1}<\epsilon_{2}$ and $\lambda$ satisfies the $\epsilon_{1}$-weak reverse doubling condition, then $\lambda$ also satisfies the $\epsilon_{2}$-weak reverse doubling condition.

(ii) Assume that $\operatorname{diam}(\mathscr{X})=\infty$. For any fixed $x \in \mathscr{X}$, we know that

$$
\lim _{r \rightarrow 0} \lambda(x, r)=0, \quad \lim _{r \rightarrow \infty} \lambda(x, r)=\infty .
$$

(ii) It is easy to see that the $\epsilon$-weak reverse doubling condition is much weaker than the assumption introduced by Bui and Duong in $[4$, Subsection 7.3]: there exists $m \in(0, \infty)$ such that, for all $x \in \mathscr{X}$ and $a, r \in(0, \infty), \lambda(x, a r)=a^{m} \lambda(x, r)$.
Definition 7 (see [14]). For any two balls $B \subset S$, define

$$
K_{B, S}=1+\int_{2 S \backslash B} \frac{1}{\lambda\left(c_{B}, d\left(x, c_{B}\right)\right)} d \mu(x),
$$

where $c_{B}$ is the center of the ball $B$.

Remark 8. The following discrete version, $\widetilde{K}_{B, S}$, of $K_{B, S}$ defined in Definition 7, was first introduced by Bui and Duong [4] in nonhomogeneous metric measure spaces, which is more close to the quantity $K_{\mathrm{Q}, R}$ introduced by Tolsa [1] in the setting of nondoubling measures. For any two balls $B \subset S$, let $\widetilde{K}_{B, S}$ be defined by

$$
\widetilde{K}_{B, S}:=1+\sum_{k=1}^{N_{B, S}} \frac{\mu\left(6^{k} B\right)}{\lambda\left(c_{B}, 6 r^{k} r_{B}\right)}
$$

where $r_{B}$ and $r_{S}$, respectively, denote the radii of the balls $B$ and $S$, and $N_{B, S}$ denotes the smallest integer satisfying $6^{N_{B, S}} r_{B} \geq r_{S}$. Obviously, $K_{B, S} \leq C \widetilde{K}_{B, S}$. As was pointed by Bui and Duong [4], in general, it is not true that $K_{B, S} \sim C \widetilde{K}_{B, S}$.

Definition 9 (see [14]). Let $\rho \in(1, \infty)$. A function $f \in L_{\text {loc }}^{1}(\mu)$ is said to be in the space $\operatorname{RBMO}(\mu)$ if there exist a positive constant $C$, and for any ball $B \subset \mathscr{X}$, a number $f_{B}$ such that

$$
\frac{1}{\mu(\rho B)} \int_{B}\left|f(x)-f_{B}\right| d \mu(x) \leq C,
$$

for any two balls $B$ and $B_{1}$ such that $B \subset B_{1}$,

$$
\left|f_{B}-f_{B_{1}}\right| \leq C K_{B, B_{1}} \text {. }
$$

The infimum of the positive constants $C$ satisfying above two inequalities is defined to be the $\operatorname{RBMO}(\mu)$ norm of $f$ and denoted by $\|f\|_{\mathrm{RBMO}(\mu)}$.

From $[14$, Lemma 4.6], it follows that the space $\operatorname{RBMO}(\mu)$ is independent of $\rho \in(1, \infty)$.

In this paper, we consider a variant of the fractional integrals from [7, Definition 4.1].

Definition 10. Let $0<\alpha<n$ and $0<\delta \leq 1$. A function $K_{\alpha} \epsilon$ $L_{\text {loc }}^{1}(\mathscr{X} \times \mathscr{X} \backslash\{(x, y): x=y\})$ is said to be a fractional kernel of order $\alpha$ and regularity $\delta$ if it satisfies the following two conditions:

(i) for all $x, y \in \mathscr{X}$ with $x \neq y$,

$$
\left|K_{\alpha}(x, y)\right| \leq C \frac{1}{[\lambda(x, d(x, y))]^{1-\alpha / n}} ;
$$

(ii) for all $x, \tilde{x}, y \in \mathscr{X}$ with $\lambda(x, d(x, y)) \geq 2 \lambda(x, d(x, \tilde{x}))$,

$$
\begin{aligned}
& \left|K_{\alpha}(x, y)-K_{\alpha}(\tilde{x}, y)\right|+\left|K_{\alpha}(y, x)-K_{\alpha}(y, \tilde{x})\right| \\
& \quad \leq C \frac{[\lambda(x, d(x, \tilde{x}))]^{\delta / n}}{[\lambda(x, d(x, y))]^{1-\alpha / n+\delta / n}} .
\end{aligned}
$$


A linear operator $I_{\alpha}$ is called fractional integral operator with $K_{\alpha}$ satisfying (10) and (11),

$$
I_{\alpha} f(x):=\int_{\mathscr{X}} K_{\alpha}(x, y) f(y) d \mu(y) .
$$

Remark 11. By taking $\lambda(x, d(x, y))=d(x, y)^{n}$, it is easy to see that Definition 10 in this paper contains Definition 4.1 introduced by García-Cuerva and Gatto in [7], and Definition 10 is similar to Definition 1.9 introduced by Fu et al. in [11].

Definition 12. Let $K_{\alpha}$ be a fractional kernel of order $\alpha$ and regularity $\delta, f \in L^{p}(\mu)$ and $0<\alpha-n / p<\delta$. We define

$$
\tilde{I}_{\alpha} f(x)=\int_{\mathscr{X}}\left\{K_{\alpha}(x, y)-K_{\alpha}\left(x_{0}, y\right)\right\} f(y) d \mu(y),
$$

where $x_{0}$ is some fixed point of $\mathscr{X}$.

We observe that the integral in (13) converges both locally and at $\infty$ as a consequence of (10), (11), and Hölder's inequality. Of course the function just defined depends on the election of $x_{0}$, but the difference between any two functions obtained in (13) for different elections of $x_{0}$ is just a constant.

From now on, we will assume that $\mu(\mathscr{X})=\infty$. The results below are also true when $\mu(\mathscr{X})<\infty$.

Now we state the first main theorem of this paper.

Theorem 13. Let $1 \leq p<n / \alpha$ and $1 / q=1 / p-\alpha / n$. If $\lambda$ satisfy the $\epsilon$-weak reverse doubling condition with $\epsilon \epsilon$ $\left(0, \min \left\{\alpha / n,(1 / p-\alpha / n) p^{\prime}\right\}\right)$, then

$$
\mu\left(\left\{x \in \mathscr{X}:\left|I_{\alpha} f(x)\right|>\nu\right\}\right) \leq\left(\frac{C\|f\|_{L^{p}(\mu)}}{\nu}\right)^{q}
$$

that is, $I_{\alpha}$ is a bounded operator from $L^{p}(\mu)$ into the space $L^{q, \infty}(\mu)$.

Next, let us introduce Lipschitz spaces on nonhomogeneous metric measure spaces.

Definition 14. Given that $\beta \in(0,1]$, we say that the function $f: X \rightarrow \mathbb{C}$ satisfies a Lipschitz condition of order $\beta$ provided that

$$
|f(x)-f(y)| \leq C[\lambda(x, d(x, y))]^{\beta / n} \quad \text { for every } x, y \in \mathscr{X}
$$

and the smallest constant in inequality (15) will be denoted by $\|f\|_{\operatorname{Lip}(\beta)}$. It is easy to see that the linear space with the norm $\|\cdot\|_{\operatorname{Lip}(\beta)}$ is a Banach space, and we will call it $\operatorname{Lip}(\beta)$.

Remark 15. Lipschitz condition can also be defined by

$$
|f(x)-f(y)| \leq C[\lambda(y, d(x, y))]^{\beta / n} \quad \text { for every } x, y \in \mathscr{X}
$$

by (2), it is easy to see that (15) and (15)' are equivalent.
The second main result of this paper is the following some equivalent characterizations for the Lipschitz spaces.

Theorem 16. For a function $f \in L_{\text {loc }}^{1}(\mu)$, the conditions $(A)$, $(B)$, and $(C)$ are equivalent as follows.

(A) There exist some constant $C_{1}$ and a collection of numbers of $f_{B}$, one for each $B$, such that these two properties hold: for any all $B$ with radius $r$

$$
\frac{1}{\mu(2 B)} \int_{B}\left|f(x)-f_{B}\right| d \mu(x) \leq C_{1} \lambda(x, r)^{\beta / n},
$$

and for any ball $U$ such that $B \in U$ and radius $(U) \leq$ $2 r$,

$$
\left|f_{B}-f_{U}\right| \leq C_{1} \lambda(x, r)^{\beta / n}
$$

(B) There is a constant $C_{2}$ such that

$$
|f(x)-f(y)| \leq C_{2} \lambda(x, d(x, y))^{\beta / n},
$$

for $\mu$-almost every $x$ and $y$ in the support of $\mu$.

(C) For any given $p, 1 \leq p \leq \infty$, there is a constant $C(p)$, such that for every ball $B$ of radius $r$, one has

$$
\left(\frac{1}{\mu(B)} \int_{B}\left|f(x)-m_{B}(f)\right|^{p} d \mu(x)\right)^{1 / p} \leq C(p) \lambda(x, r)^{\beta / n}
$$

where $m_{B}=(1 / \mu(B)) \int_{B} f(y) d \mu(y)$ and also for any ball $U$ such that $B \subset U$ and radius $(U) \leq 2 r$

$$
\left|m_{B}(f)-m_{U}(f)\right| \leq C(p) \lambda(x, r)^{\beta / n} .
$$

In addition, the quantities inf $C_{1}$, inf $C_{2}$, and inf $C(p)$ with a fixed $p$ are equivalent.

Now we state the third main result of this paper.

Theorem 17. Let $K_{\alpha}$ be a fractional kernel. $n / \alpha<p \leq \infty$ and $\alpha-n / p<\delta$. If $\lambda$ satisfy the $\epsilon$-weak reverse doubling condition with $\epsilon \in\left(0, \min \left\{(1-((\alpha-\delta) / n)) p^{\prime},(\alpha / n-1 / p) p^{\prime}\right\}\right)$, then $\widetilde{I}_{\alpha}$ maps $L^{p}(\mu)$ boundedly into $\operatorname{Lip}(\alpha-n / p)$.

Theorem 18. Let $K_{\alpha}$ be a fractional kernel and $\alpha+\beta<\delta$; if $\lambda$ satisfy the $\epsilon$-weak reverse doubling condition with $\epsilon \epsilon$ $(0, \min \{(\alpha+\beta) / n,(\delta-\alpha-\beta) / n\})$, then $\widetilde{I}_{\alpha} \operatorname{maps} \operatorname{Lip}(\beta)$ boundedly into $\operatorname{Lip}(\alpha+\beta)$ if and only if $\widetilde{I}_{\alpha}(1)=0$.

Finally we present a result which can be viewed either as an extension of the case $p=\infty$ of the Theorem 17 or as extension of the case $\beta=0$ of Theorem 18 .

Theorem 19. Let $K_{\alpha}$ be a fractional kernel and $0<\alpha<\delta$. If $\lambda$ satisfy the $\epsilon$-weak reverse doubling condition with $\epsilon \epsilon$ $(0, \min \{\alpha / n,(\delta-\alpha) / n\})$, then $\widetilde{I}_{\alpha}$ maps $R B M O(\mu)$ boundedly into $\operatorname{Lip}(\alpha)$ if and only if $\widetilde{I}_{\alpha}(1)=0$.

Finally, we make some conventions on notation. Throughout the whole paper, $C$ stands for a positive constant, which is independent of the main parameters, but it may vary from line to line. 


\section{Proof of Theorem 13}

Proof of Theorem 13. We are going to adapt to our context of the proof given by García-Cuerva and Gatto [7]. Consider

$$
\begin{aligned}
\left|I_{\alpha} f(x)\right| \leq & \int_{\mathscr{X}} \frac{|f(y)|}{[\lambda(x, d(x, y))]^{1-\alpha / n}} d \mu(y) \\
\leq & \int_{B(x, r)} \frac{|f(y)|}{[\lambda(x, d(x, y))]^{1-\alpha / n}} d \mu(y) \\
& +\int_{\mathscr{X} \backslash B(x, r)} \frac{|f(y)|}{[\lambda(x, d(x, y))]^{1-\alpha / n}} d \mu(y) \\
:= & I_{1}+I_{2} .
\end{aligned}
$$

By Hölder's inequality, if $p>1$, then $1-(1-\alpha / n) p^{\prime}<0$;

$$
\begin{aligned}
& \left|I_{2}\right| \leq\|f\|_{L^{p}(\mu)} \\
& \times\left(\int_{\mathscr{X} \backslash B(x, r)} \frac{1}{[\lambda(x, d(x, y))]^{(1-\alpha / n) p^{\prime}}} d \mu(y)\right)^{1 / p^{\prime}} \\
& \leq\|f\|_{L^{p}(\mu)} \\
& \times\left(\sum_{j=1}^{\infty} \int_{2^{j} B \backslash 2^{j-1} B} \frac{1}{[\lambda(x, d(x, y))]^{(1-\alpha / n) p^{\prime}}} d \mu(y)\right)^{1 / p^{\prime}} \\
& \leq\|f\|_{L^{p}(\mu)} \\
& \times\left(\sum_{j=1}^{\infty} \frac{\mu\left(B\left(x, 2^{j} r\right)\right)}{\left[\lambda\left(x, 2^{j-1} r\right)\right]^{(1-\alpha / n) p^{\prime}}}\right)^{1 / p^{\prime}} \\
& \leq\|f\|_{L^{p}(\mu)} \\
& \times\left(\sum_{j=1}^{\infty} \frac{\lambda\left(x, 2^{j} r\right)}{\left[\lambda\left(x, 2^{j-1} r\right)\right]^{(1-\alpha / n) p^{\prime}}}\right)^{1 / p^{\prime}} \\
& \leq C\|f\|_{L^{p}(\mu)}\left(\sum_{j=1}^{\infty}\left[\lambda\left(x, 2^{j-1} r\right)\right]^{1-(1-\alpha / n) p^{\prime}}\right)^{1 / p^{\prime}} \\
& \leq C\|f\|_{L^{p}(\mu)}\left(\sum_{j=1}^{\infty} \frac{1}{\left[C\left(2^{j}\right)\right]^{(1-\alpha / n) p^{\prime}-1}}\right)^{1 / p^{\prime}} \\
& \times[\lambda(x, r)]^{\alpha / n-1 / p} \\
& \leq C\|f\|_{L^{p}(\mu)}[\lambda(x, r)]^{\alpha / n-1 / p},
\end{aligned}
$$

which holds even for $p=1$. We can and assume that $\|f\|_{L^{p}(\mu)}=1$. By (5), we can choose $r \in(0, \infty)$ such that $C[\lambda(x, r)]^{\alpha / n-1 / p}=v / 2$. Then

$$
\begin{aligned}
\left\{x \in \mathscr{X}:\left|I_{\alpha} f(x)\right|>\nu\right\} & \subset\left\{x \in \mathscr{X}:\left|I_{1}\right|>\frac{\nu}{2}\right\} \\
& \cup\left\{x \in \mathscr{X}:\left|I_{2}\right|>\frac{\nu}{2}\right\} .
\end{aligned}
$$

By the relation between $r$ and $\nu, \mu\left(\left\{x \in \mathscr{X}:\left|I_{2}\right|>\nu / 2\right\}\right)=0$. We use Hölder's inequality once more to obtain

$$
\left|I_{1}\right| \leq\left(\int_{B(x, r)} \frac{d \mu(y)}{[\lambda(x, d(x, y))]^{1-\alpha / n}}\right)^{1 / p^{\prime}}
$$

$$
\times\left(\int_{B(x, r)} \frac{|f(y)|^{p}}{[\lambda(x, d(x, y))]^{1-\alpha / n}} d \mu(y)\right)^{1 / p}
$$

$$
\begin{aligned}
& \leq C\left(\sum_{j=0}^{\infty} \frac{\mu\left(B\left(x, 2^{-j} r\right)\right)}{\left[\lambda\left(x, 2^{-j-1} r\right)\right]^{1-\alpha / n}}\right)^{1 / p^{\prime}} \\
& \quad \times\left(\int_{B(x, r)} \frac{|f(y)|^{p}}{[\lambda(x, d(x, y))]^{1-\alpha / n}} d \mu(y)\right)^{1 / p}
\end{aligned}
$$$$
\leq C\left(\sum_{j=0}^{\infty} \frac{\lambda\left(x, 2^{j} r\right)}{\left[\lambda\left(x, 2^{-j-1} r\right)\right]^{1-\alpha / n}}\right)^{1 / p^{\prime}}
$$

$$
\times\left(\int_{B(x, r)} \frac{|f(y)|^{p}}{[\lambda(x, d(x, y))]^{1-\alpha / n}} d \mu(y)\right)^{1 / p}
$$$$
\leq C\left(\sum_{j=0}^{\infty}\left[\lambda\left(x, 2^{-j-1} r\right)\right]^{\alpha / n}\right)^{1 / p^{\prime}}
$$

$$
\times\left(\int_{B(x, r)} \frac{|f(y)|^{p}}{[\lambda(x, d(x, y))]^{1-\alpha / n}} d \mu(y)\right)^{1 / p}
$$

$$
\leq C\left(\sum_{j=0}^{\infty} \frac{1}{\left[C\left(2^{j}\right)\right]^{\alpha / n}}[\lambda(x, r)]^{\alpha / n}\right)^{1 / p^{\prime}}
$$

$$
\times\left(\int_{B(x, r)} \frac{|f(y)|^{p}}{[\lambda(x, d(x, y))]^{1-\alpha / n}} d \mu(y)\right)^{1 / p}
$$$$
\leq C[\lambda(x, r)]^{\alpha / p^{\prime} n}
$$

$$
\times\left(\int_{B(x, r)} \frac{|f(y)|^{p}}{[\lambda(x, d(x, y))]^{1-\alpha / n}} d \mu(y)\right)^{1 / p} .
$$


Then, by applying Tchebichev's inequality, we have

$$
\begin{aligned}
& \mu\left(\left\{x \in \mathscr{X}:\left|I_{\alpha} f(x)\right|>v\right\}\right) \\
& \leq \mu\left(\left\{x \in \mathscr{X}:\left|I_{1}\right|>\frac{v}{2}\right\}\right) \\
& \leq C[\lambda(x, r)]^{\alpha p / p^{\prime} n} v^{-p} \\
& \times \int_{\mathscr{X}} \int_{B(x, r)} \frac{|f(y)|^{p}}{[\lambda(x, d(x, y))]^{1-\alpha / n}} d \mu(y) d \mu(x) \\
&= C[\lambda(x, r)]^{\alpha p / p^{\prime} n} v^{-p} \\
& \times \int_{\mathscr{X}} \int_{B(y, r)} \frac{d \mu(x)}{[\lambda(x, d(x, y))]^{1-\alpha / n}}|f(y)|^{p} d \mu(y) \\
& \leq C[\lambda(x, r)]^{\alpha p / p^{\prime} n} v^{-p}[\lambda(x, r)]^{\alpha / n}\|f\|_{L^{p}(\mu)} \\
&= C[\lambda(x, r)]=C v^{-q} .
\end{aligned}
$$

This completes the proof of Theorem 13.

Corollary 20. Let $1<p<n / \alpha$ and $1 / q=1 / p-\alpha / n$. If $\lambda$ satisfy the $\epsilon$-weak reverse doubling condition with $\epsilon \epsilon$ $\left(0, \min \left\{\alpha / n,(1 / p-\alpha / n) p^{\prime}\right\}\right)$, then

$$
\left\|I_{\alpha} f\right\|_{L^{q}(\mu)} \leq C\|f\|_{L^{p}(\mu)} .
$$

Proof. It suffices to apply Marcinkiewicz's interpolation theorem with indices slightly bigger and slightly smaller than p.

\section{Proof of Theorem 16}

Before we give the proof of Theorem 16, we first introduce a technical lemma from [8, Lemma 3.2].

Lemma 21. Let $f \in L_{\text {loc }}^{1}(\mu)$. If $\beta_{2}>2^{d}$, then, for almost every $x$ with respect to $\mu$, there exists a sequence of $\left(2, \beta_{2}\right)$-doubling balls $B_{j}=B\left(x, r_{j}\right)$ with $r_{j} \rightarrow 0$, such that

$$
\lim _{j \rightarrow \infty} \frac{1}{\mu\left(B_{j}\right)} \int_{B_{j}} f(y) d \mu(y)=f(x) .
$$

Proof of Theorem 16. (A) $\Rightarrow$ (B). Consider $x$ as in the lemma and let $B_{j}=B\left(x, r_{j}\right), j \geq 1$, a sequence of $\left(2, \beta_{2}\right)$-doubling balls with $r_{j} \rightarrow 0$. Consider

$$
\begin{aligned}
\left|m_{B_{j}}(f)-f_{B_{j}}\right| & \leq \frac{1}{\mu\left(B_{j}\right)} \int_{B_{j}}\left|f(y)-f_{B_{j}}\right| d \mu(y) \\
& \leq \frac{\mu\left(2 B_{j}\right)}{\mu\left(B_{j}\right)} \frac{1}{\mu\left(2 B_{j}\right)} \int_{B_{j}}\left|f(y)-f_{B_{j}}\right| d \mu(y) \\
& \leq \beta C_{1}\left[\lambda\left(x, r_{j}\right)\right]^{\beta / n},
\end{aligned}
$$

and by (5), Lemma 21, we obtain that

$$
\lim _{j \rightarrow \infty} f_{B_{j}}=f(x) .
$$

Let $x$ and $y$ be two points as in the lemma; take $B=B(x, r)$ any ball with $r \leq d(x, y)$ and let $U=B(x, 2 d(x, y))$. Now define $B_{k}=B\left(x, 2^{k} r\right)$, for $0 \leq k \leq \widetilde{k}$, where $\widetilde{k}$ is the first integer such that $2^{\tilde{k}} r \geq d(x, y)$. Then

$$
\begin{aligned}
\left|f_{B}-f_{U}\right| & \leq \sum_{k=0}^{\tilde{k}-1}\left|f_{B_{k}}-f_{B_{k+1}}\right|+\left|f_{B_{\tilde{k}}}-f_{U}\right| \\
& \leq C_{1} \sum_{k=0}^{\tilde{k}}\left[\lambda\left(x, 2^{k} r\right)\right]^{\beta / n} \\
& \leq C_{1} \sum_{k=0}^{\tilde{k}} c_{\lambda}^{\beta k / n}[\lambda(x, r)]^{\beta / n} \\
& \leq C^{\prime} C_{1} \lambda(x, d(x, y))^{\beta / n},
\end{aligned}
$$

where $C^{\prime}$ is independent of $x$ and $d(x, y)$.

A similar argument can be made for the point $y$ with any ball $B^{\prime}=B(y, s)$ such that $s \leq d(x, y)$ and $V=B(y, 3 d(x, y))$. Therefore

$$
\begin{aligned}
\left|f_{B}-f_{B^{\prime}}\right| & \leq\left|f_{B}-f_{U}\right|+\left|f_{U}-f_{V}\right|+\left|f_{V}-f_{B^{\prime}}\right| \\
& \leq C^{\prime \prime} C_{1} \lambda(x, d(x, y))^{\beta / n} .
\end{aligned}
$$

Take two sequences of $\left(2, \beta_{2}\right)$-doubling balls $B_{j}=B\left(x, r_{j}\right)$ and $B^{\prime}=B\left(y, s_{j}\right)$ with $r_{j} \rightarrow 0$ and $s_{j} \rightarrow 0$. We have

$$
|f(x)-f(y)|=\lim _{j \rightarrow 0}\left|f_{B_{j}}-f_{B_{j}^{\prime}}\right| \leq C^{\prime \prime} C_{1} \lambda(x, d(x, y))^{\beta / n} .
$$

(B) $\Rightarrow(\mathrm{C})$. For $x_{0} \in B=B(x, r)$, by the properties of function $\lambda$ and Hölder's inequality, we obtain

$$
\begin{aligned}
& \left(\frac{1}{\mu(B)} \int_{B}\left|f(x)-m_{B}(f)\right|^{p} d \mu(x)\right)^{1 / p} \\
& \quad \leq\left(\frac{1}{\mu(B)} \int_{B}\left|\frac{1}{\mu(B)} \int_{B}(f(x)-f(y)) d \mu(y)\right|^{p} d \mu(x)\right)^{1 / p} \\
& \quad \leq\left(\frac{1}{\mu(B)} \int_{B}\left[C_{2} \lambda(x, r)\right]^{\beta p / n} d \mu(x)\right)^{1 / p} \\
& \quad \leq\left(\frac{1}{\mu(B)} \int_{B}\left[C_{2} C_{\lambda} \lambda\left(x_{0}, r\right)\right]^{\beta p / n} d \mu(x)\right)^{1 / p} \\
& \quad \leq C_{2} C_{\lambda}\left[\lambda\left(x_{0}, r\right)\right]^{\beta / n} \leq C_{2} C_{\lambda}^{2}[\lambda(x, r)]^{\beta / n} .
\end{aligned}
$$

By the similar argument, for any ball $U$ such that $B \subset U$ and radius $U \leq 2 r$,

$$
\begin{aligned}
\left|m_{B}(f)-m_{U}(f)\right| & \leq C_{2} C_{\lambda}^{2}[\lambda(x, 2 r)]^{\beta / n} \\
& \leq c_{\lambda}^{\beta / n} C_{2} C_{\lambda}^{2}[\lambda(x, r)]^{\beta / n} .
\end{aligned}
$$


$(\mathrm{C}) \Rightarrow(\mathrm{A})$. Define first $f_{B}=m_{B}(f)$. Then (17) is exactly (20). To prove (16), we write

$$
\begin{aligned}
& \frac{1}{\mu(2 B)} \int_{B}\left|f(x)-f_{B}\right| d \mu(x) \\
& \quad \leq \frac{1}{\mu(2 B)}\left(\int_{B}\left|f(x)-f_{B}\right|^{p} d \mu(x)\right)^{1 / p} \mu(B)^{1 / p^{\prime}} \\
& \quad \leq \frac{\mu(B)}{\mu(2 B)}\left(\frac{1}{\mu(B)} \int_{B}\left|f(x)-f_{B}\right|^{p} d \mu(x)\right)^{1 / p} \\
& \leq C(p) \lambda(x, r)^{\beta / n} .
\end{aligned}
$$

This concludes the proof of the theorem.

Remark 22. Theorem 16 is also true if the number 2 in condition (A) is replaced by any fixed $\rho>1$. In that case, the proof uses $\left(\rho, \beta_{\rho}\right)$-doubling balls, that is, balls satisfying $\mu(\rho B) \leq \beta_{\rho} \mu(B)$.

\section{Proofs of Theorems 17-19}

Proof of Theorem 17. Without loss of generality, we assume that $p<\infty$. Consider $x \neq y$ and let $B$ be the ball with center $x$ and radius $r=d(x, y)$. Then, we have

$$
\begin{aligned}
\left|\tilde{I}_{\alpha} f(x)-\tilde{I}_{\alpha} f(y)\right| \leq & \int_{2 B}\left|K_{\alpha}(x, z)\right||f(z)| d \mu(z) \\
& +\int_{2 B}\left|K_{\alpha}(y, z)\right||f(z)| d \mu(z) \\
& +\int_{\mathscr{X} \backslash 2 B}\left|K_{\alpha}(x, z)-K_{\alpha}(y, z)\right| \\
& \times|f(z)| d \mu(z) \\
:= & J_{1}+J_{2}+J_{3} .
\end{aligned}
$$

For the first term, by $n / \alpha<p$, then $1-(1-\alpha / n) p^{\prime}>0$,

$$
\begin{aligned}
J_{1} & \leq \int_{2 B} \frac{|f(z)|}{[\lambda(x, d(x, y))]^{1-\alpha / n}} d \mu(z) \\
& \leq\|f\|_{L^{p}(\mu)}\left(\int_{2 B} \frac{d \mu(z)}{[\lambda(x, d(x, y))]^{(1-\alpha / n) p^{\prime}}}\right)^{1 / p^{\prime}} \\
& \leq\|f\|_{L^{p}(\mu)}\left(\sum_{j=0}^{\infty} \frac{\mu\left(B\left(x, 2^{-j+1} r\right)\right)}{\left[\lambda\left(x, d\left(x, 2^{-j} r\right)\right)\right]^{(1-\alpha / n) p^{\prime}}}\right)^{1 / p^{\prime}} \\
& \leq\|f\|_{L^{p}(\mu)}\left(\sum_{j=0}^{\infty} \frac{\lambda\left(x, 2^{-j+1} r\right)}{\left[\lambda\left(x, d\left(x, 2^{-j} r\right)\right)\right]^{(1-\alpha / n) p^{\prime}}}\right)^{1 / p^{\prime}} \\
& \leq C\|f\|_{L^{p}(\mu)}\left(\sum_{j=0}^{\infty}\left[\lambda\left(x, d\left(x, 2^{-j} r\right)\right)\right]^{1-(1-\alpha / n) p^{\prime}}\right)^{1 / p^{\prime}}
\end{aligned}
$$

$$
\begin{aligned}
& \leq C\|f\|_{L^{p}(\mu)}\left(\sum_{j=0}^{\infty} \frac{1}{\left[C\left(2^{j}\right)\right]^{(\alpha / n-1 / p) p^{\prime}}}\right)^{1 / p^{\prime}} \\
& \quad \times[\lambda(x, r)]^{\alpha / n-1 / p} \\
& \leq C\|f\|_{L^{p}(\mu)}[\lambda(x, r)]^{\alpha / n-1 / p} .
\end{aligned}
$$

The second term is estimated in a similar way after noting that $2 B \subset B(y, 3 r)$.

Next, by using (2), Hölder's inequality, and $\alpha-n / p<\delta$, we get

$$
\begin{aligned}
J_{3} \leq & C \int_{\mathscr{X} \backslash 2 B} \frac{[\lambda(x, d(x, y))]^{\delta / n}}{[\lambda(x, d(x, z))]^{1-\alpha / n+\delta / n}}|f(z)| d \mu(z) \\
\leq & C[\lambda(x, d(x, y))]^{\delta / n}\|f\|_{L^{p}(\mu)} \\
& \times\left(\int_{\mathscr{X} \backslash 2 B} \frac{1}{[\lambda(x, d(x, z))]^{(1-(\alpha-\delta) / n) p^{\prime}}} d \mu(z)\right)^{1 / p^{\prime}} \\
\leq & C\|f\|_{L^{p}(\mu)}[\lambda(x, d(x, y))]^{\delta / n} \\
& \times\left(\int_{X^{\prime}(2 B} \frac{1}{[\lambda(x, d(x, z))]^{(1-(\alpha-\delta) / n) p^{\prime}}} d \mu(z)\right)^{1 / p^{\prime}} \\
\leq & C\|f\|_{L^{p}(\mu)}[\lambda(x, d(x, y))]^{\delta / n}[\lambda(x, d(x, y))]^{(\alpha-\delta) / n-1 / p} \\
\leq & C\|f\|_{L^{p}(\mu)}[\lambda(x, d(x, y))]^{\alpha / n-1 / p} .
\end{aligned}
$$

Putting together the three estimates,

$$
\left|\widetilde{I}_{\alpha} f(x)-\widetilde{I}_{\alpha} f(y)\right| \leq C\|f\|_{L^{p}(\mu)}[\lambda(x, d(x, y))]^{\alpha / n-1 / p}
$$

then $\widetilde{I}_{\alpha}$ maps $L^{p}(\mu)$ boundedly into $\operatorname{Lip}(\alpha-n / p)$.

Proof of Theorem 18. If $\widetilde{I}_{\alpha} \in \operatorname{Lip}(\beta)$, then by the continuity of the operator $\widetilde{I}_{\alpha}$ implies that $\widetilde{I}_{\alpha}(1)$ must be constant; that is, $\tilde{I}_{\alpha}(1)(x)=\tilde{I}_{\alpha}(1)\left(x_{0}\right)=0$.

On the other hand, we can observe that

$$
\widetilde{I}_{\alpha}(1)=0 \Longleftrightarrow \widetilde{I}_{\alpha}(1)(x)-\widetilde{I}_{\alpha}(1)(y)=0 ;
$$

this implies that

$$
\int_{X}\left\{K_{\alpha}(x, z)-K_{\alpha}(y, z)\right\} d \mu(z)=0
$$


Thus we can write

$$
\begin{aligned}
& \widetilde{I}_{\alpha}(f)(x)-\widetilde{I}_{\alpha}(f)(y) \\
& =\int_{X}\left\{K_{\alpha}(x, z)-K_{\alpha}(y, z)\right\}(f(z)-f(x)) d \mu(z) \\
& =\int_{2 B(x, r)} K_{\alpha}(x, z)(f(z)-f(x)) d \mu(z) \\
& \quad+\int_{2 B(x, r)}-K_{\alpha}(y, z)(f(z)-f(x)) d \mu(z) \\
& \quad+\int_{X \backslash 2 B(x, r)}\left\{K_{\alpha}(x, z)-K_{\alpha}(y, z)\right\} \\
& \quad \times(f(z)-f(x)) d \mu(z) \\
& :=M_{1}+M_{2}+M_{3},
\end{aligned}
$$

where $r=d(x, y)$. For $M_{1}$,

$$
\begin{aligned}
\left|M_{1}\right| & \leq \int_{2 B(x, r)} \frac{|f(z)-f(x)|}{[\lambda(x, d(x, z))]^{1-\alpha / n}} d \mu(z) \\
& \leq C \int_{2 B(x, r)} \frac{[\lambda(x, d(x, z))]^{\beta / n}}{[\lambda(x, d(x, z))]^{1-\alpha / n}} d \mu(z) \\
& \leq C[\lambda(x, d(x, y))]^{(\alpha+\beta) / n} .
\end{aligned}
$$

Similarly, we know that $2 B(x, r) \subset 3 B(y, r)$; using Hölder's inequality $\left(1 / t+1 / t^{\prime}=1\right)$, and letting $t>n / \alpha$, then we have

$\left|M_{2}\right|$

$\leq \int_{2 B(x, r)} \frac{|f(z)-f(x)|}{[\lambda(y, d(y, z))]^{1-\alpha / n}} d \mu(z)$

$\leq C \int_{2 B(x, r)} \frac{[\lambda(x, d(x, z))]^{\beta / n}}{[\lambda(y, d(y, z))]^{1-\alpha / n}} d \mu(z)$

$\leq C\left(\int_{2 B(x, r)}[\lambda(x, d(x, z))]^{\beta t / n} d \mu(z)\right)^{1 / t}$

$\times\left(\int_{2 B(x, r)} \frac{1}{[\lambda(y, d(y, z))]^{(1-\alpha / n) t^{\prime}}} d \mu(z)\right)^{1 / t^{\prime}}$

$\leq C\left(\sum_{j=0}^{\infty} \int_{2^{-j+1} B(x, r) \backslash 2^{-j} B(x, r)}[\lambda(x\right.$,

$\left.\left.\left.d\left(x, 2^{-j+1} r\right)\right)\right]^{\beta t / n} d \mu(z)\right)^{1 / t}$

$\times\left(\int_{3 B(y, r)} \frac{1}{[\lambda(y, d(y, z))]^{(1-\alpha / n) t^{\prime}}} d \mu(z)\right)^{1 / t^{\prime}}$ $\leq C[\lambda(x, d(x, y))]^{\alpha / n}[\lambda(y, d(x, y))]^{\beta / n}$

$\leq C[\lambda(x, d(x, y))]^{(\alpha+\beta) / n}$.

In order to estimate $M_{3}$, we use (2) to obtain

$$
\begin{aligned}
& \left|M_{3}\right| \\
& \leq C \int_{\mathscr{X} \backslash 2 B(x, r)} \frac{[\lambda(x, d(x, y))]^{\delta / n}[\lambda(x, d(x, z))]^{\beta / n}}{[\lambda(x, d(x, z))]^{1-(\alpha-\delta) / n}} d \mu(z) \\
& \leq C[\lambda(x, d(x, y))]^{\delta / n} \\
& \quad \times \int_{\mathscr{X} \backslash 2 B(x, r)[\lambda(x, d(x, z))]^{1-(\alpha+\beta-\delta) / n}} \frac{1 \mu(z)}{\leq} C[\lambda(x, d(x, y))]^{\delta / n}[\lambda(x, d(x, y))]^{(\alpha+\beta-\delta) / n} \\
& \leq C[\lambda(x, d(x, y))]^{(\alpha+\beta) / n} .
\end{aligned}
$$

Combining the estimates for $M_{1}, M_{2}$, and $M_{3}$, we obtain

$$
\left|\widetilde{I}_{\alpha} f(x)-\widetilde{I}_{\alpha} f(y)\right| \leq C[\lambda(x, d(x, y))]^{(\alpha+\beta) / n}
$$

this finishes the proof.

Proof of Theorem 19. $\widetilde{I}_{\alpha}(f) \in \operatorname{Lip}(\alpha)$ implies that $\widetilde{I}_{\alpha}(1)=0$ and, equivalently, that

$$
\int_{\mathscr{X}}\left(K_{\alpha}(x, y)-K_{\alpha}\left(x_{0}, y\right)\right) d \mu(y)=0
$$

for all $x$.

Take two points $x \neq y$ and let $B=B(x, r)$ with $r=d(x, y)$. Then

$$
\begin{aligned}
& \left|\widetilde{I}_{\alpha}(f)(x)-\widetilde{I}_{\alpha}(f)(y)\right| \\
& =\left|\int_{\mathscr{X}}\left\{K_{\alpha}(x, z)-K_{\alpha}(y, z)\right\}\left(f(z)-f_{2 B}\right) d \mu(z)\right| \\
& \leq\left|\int_{2 B} K_{\alpha}(x, z)\left(f(z)-f_{2 B}\right) d \mu(z)\right| \\
& \quad+\left|\int_{2 B} K_{\alpha}(y, z)\left(f(z)-f_{2 B}\right) d \mu(z)\right| \\
& \quad+\left|\int_{\mathscr{X} \backslash 2 B}\left\{K_{\alpha}(x, z)-K_{\alpha}(y, z)\right\}\left(f(z)-f_{2 B}\right) d \mu(z)\right| \\
& :=N_{1}+N_{2}+N_{3} .
\end{aligned}
$$


For the first term, by Hölder's inequality with some $p>n / \alpha$,

$$
\begin{aligned}
N_{1} \leq & \int_{2 B} \frac{1}{[\lambda(x, d(x, z))]^{1-\alpha / n}}\left|f(z)-f_{2 B}\right| d \mu(z) \\
\leq & \left(\int_{2 B} \frac{d \mu(z)}{[\lambda(x, d(x, z))]^{(1-\alpha / n) p^{\prime}}}\right)^{1 / p^{\prime}} \\
& \times\left(\int_{2 B}\left|f(z)-f_{2 B}\right|^{p} d \mu(z)\right)^{1 / p} \\
\leq & C[\lambda(x, r)]^{\alpha / n-1 / p} \mu(\rho B)^{1 / p}\|f\|_{\operatorname{RBMO}(\mu)} \\
\leq & C[\lambda(x, r)]^{\alpha / n}\|f\|_{\mathrm{RBMO}(\mu)} .
\end{aligned}
$$

Using $2 B \subset B(y, 3 r)$, the second term can be dealt with in the same way as the $N_{1}$; then $N_{2} \leq C[\lambda(x, r)]^{\alpha / n}\|f\|_{\mathrm{RBMO}(\mu)}$. It is easy to see that $\left|f_{2^{k+1} B}-f_{2 B}\right| \leq\|f\|_{\mathrm{RBMO}(\mu)} K_{2 B, 2^{k+1} B} \leq$ $k\|f\|_{\mathrm{RBMO}(\mu)}$; then

$$
\begin{aligned}
N_{3} \leq & \int_{X \backslash 2 B} \frac{[\lambda(x, d(x, y))]^{\delta / n}}{[\lambda(x, d(x, z))]^{1-\alpha / n+\delta / n}}\left|f(z)-f_{2 B}\right| d \mu(z) \\
\leq & C[\lambda(x, d(x, y))]^{\delta / n} \\
& \times \sum_{k=1}^{\infty}\left(\int_{2^{k+1} B \backslash 2^{k} B} \frac{\left|f(z)-f_{2^{k+1} B}\right|}{[\lambda(x, d(x, z))]^{1-\alpha / n+\delta / n}} d \mu(z)\right. \\
\leq & C[\lambda(x, d(x, y))]^{\delta / n}\|f\|_{\mathrm{RBMO}(\mu)} \\
& \left.+\frac{\left|f_{2^{k+1} B}-f_{2 B}\right|}{\left[\lambda\left(x, d\left(x, 2^{k} r\right)\right)\right]^{1-\alpha / n+\delta / n}} \mu\left(2^{k+1} B\right)\right) \\
\leq & C[\lambda(x, d(x, y))]^{\alpha / n}\|f\|_{\mathrm{RBMO}(\mu)}^{\infty} \frac{\mu\left(\rho 2^{k+1} B\right)}{\left[\lambda\left(x, d\left(x, 2^{k} r\right)\right)\right]^{1-\alpha / n+\delta / n}} \\
\leq & C[\lambda(x, d(x, y))]^{\delta / n}\|f\|_{\mathrm{RBMO}(\mu)} \\
& \left.+\sum_{k=1}^{\infty} k \frac{\mu\left(2^{k+1} B\right)}{\left[\lambda\left(x, d\left(x, 2^{k} r\right)\right)\right]^{1-\alpha / n+\delta / n}}\right) \\
(k+1)\left[\lambda\left(x, d\left(x, 2^{k} r\right)\right)\right]^{\alpha / n-\delta / n} &
\end{aligned}
$$

Thus the proof of Theorem 19 is completed.

\section{Conflict of Interests}

The authors declare that there is no conflict of interests regarding the publication of this paper.

\section{Acknowledgments}

Jiang Zhou is supported by the National Science Foundation of China (Grants nos. 11261055 and 11161044) and by the National Natural Science Foundation of Xinjiang (Grants nos. 2011211A005 and BS120104).

\section{References}

[1] X. Tolsa, "BMO, $H^{1}$, and Calderón-Zygmund operators for non doubling measures," Mathematische Annalen, vol. 319, no. 1, pp. 89-149, 2001.

[2] X. Tolsa, "Littlewood-Paley theory and the T(1) theorem with non-doubling measures," Advances in Mathematics, vol. 164, no. 1, pp. 57-116, 2001.

[3] X. Tolsa, "The space $H^{1}$ for nondoubling measures in terms of a grand maximal operator," Transactions of the American Mathematical Society, vol. 355, no. 1, pp. 315-348, 2003.

[4] T. A. Bui and X. T. Duong, "Hardy spaces, regularized BMO spaces and the boundedness of Calderón-Zygmund operators on non-homogeneous spaces," Journal of Geometric Analysis, vol. 23, no. 2, pp. 895-932, 2013.

[5] T. Hytönen, S. Liu, D. Yang, and D. Yang, "Boundedness of Calderón-Zygmund operators on non-homogeneous metric measure spaces," Canadian Journal of Mathematics, vol. 64, no. 4, pp. 892-923, 2012.

[6] T. Hytönen, D. Yang, and D. Yang, "The Hardy space $H^{1}$ on nonhomogeneous metric spaces," Mathematical Proceedings of the Cambridge Philosophical Society, vol. 153, no. 1, pp. 9-31, 2012.

[7] J. García-Cuerva and A. E. Gatto, "Boundedness properties of fractional integral operators associated to non-doubling measures," Studia Mathematica, vol. 162, no. 3, pp. 245-261, 2004.

[8] J. García-Cuerva and A. E. Gatto, "Lipschitz spaces and Calderón-Zygmund operators associated to non-doubling measures," Publicacions Matemàtiques, vol. 49, no. 2, pp. 285-296, 2005.

[9] Y. Cao and J. Zhou, "Morrey spaces for non-homogeneous metric measure spaces," Abstract and Applied Analysis, vol. 2013, Article ID 196459, 8 pages, 2013.

[10] X. Fu, D. Yang, and W. Yuan, "Boundedness of multilinear commutators of Calderón-Zygmund operators on Orlicz spaces over non-homogeneous spaces," Taiwanese Journal of Mathematics, vol. 16, no. 6, pp. 2203-2238, 2012.

[11] X. Fu, D. Yang, and W. Yuan, "Generalized fractional integrals and their commutators over non-homogeneous spaces," Taiwanese Journal of Mathematics, vol. 18, no. 2, pp. 509-557, 2014.

[12] H. Lin and D. Yang, "Equivalent boundedness of Marcinkiewicz integrals on non-homogeneous metric measure spaces," Science China. Mathematics, vol. 57, no. 1, pp. 123-144, 2014.

[13] X. Tolsa, "Painlevé's problem and the semiadditivity of analytic capacity" Acta Mathematica, vol. 190, no. 1, pp. 105-149, 2003.

[14] T. Hytönen, "A framework for non-homogeneous analysis on metric spaces, and the RBMO space of Tolsa," Publicacions Matemàtiques, vol. 54, no. 2, pp. 485-504, 2010.

[15] R. R. Coifman and G. Weiss, Analyse Harmonique Non-Commutative sur Certains Espaces Homogènes, vol. 242 of Lecture Notes in Mathematics, Springer, Berlin, Germany, 1971. 


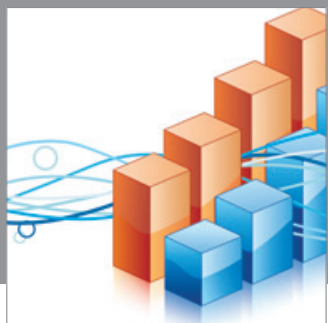

Advances in

Operations Research

mansans

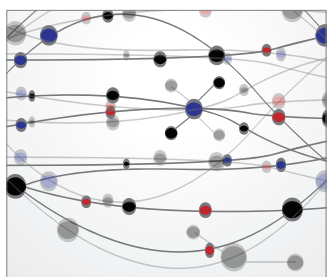

The Scientific World Journal
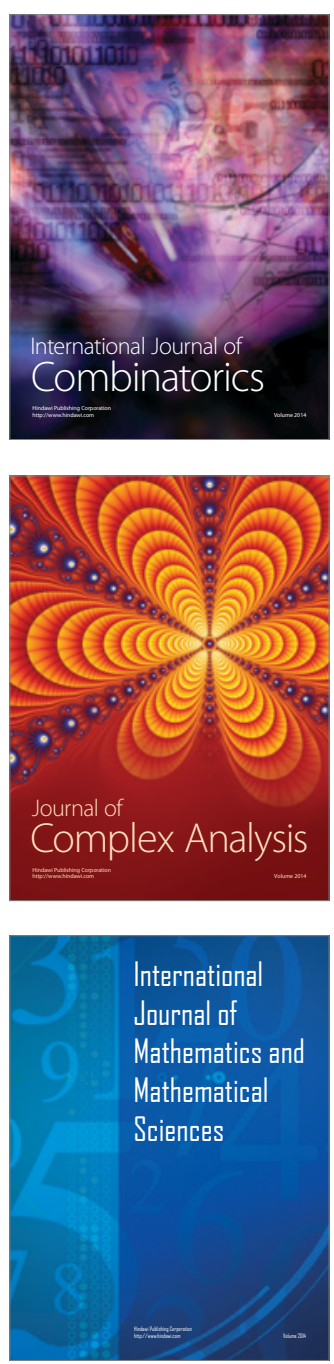
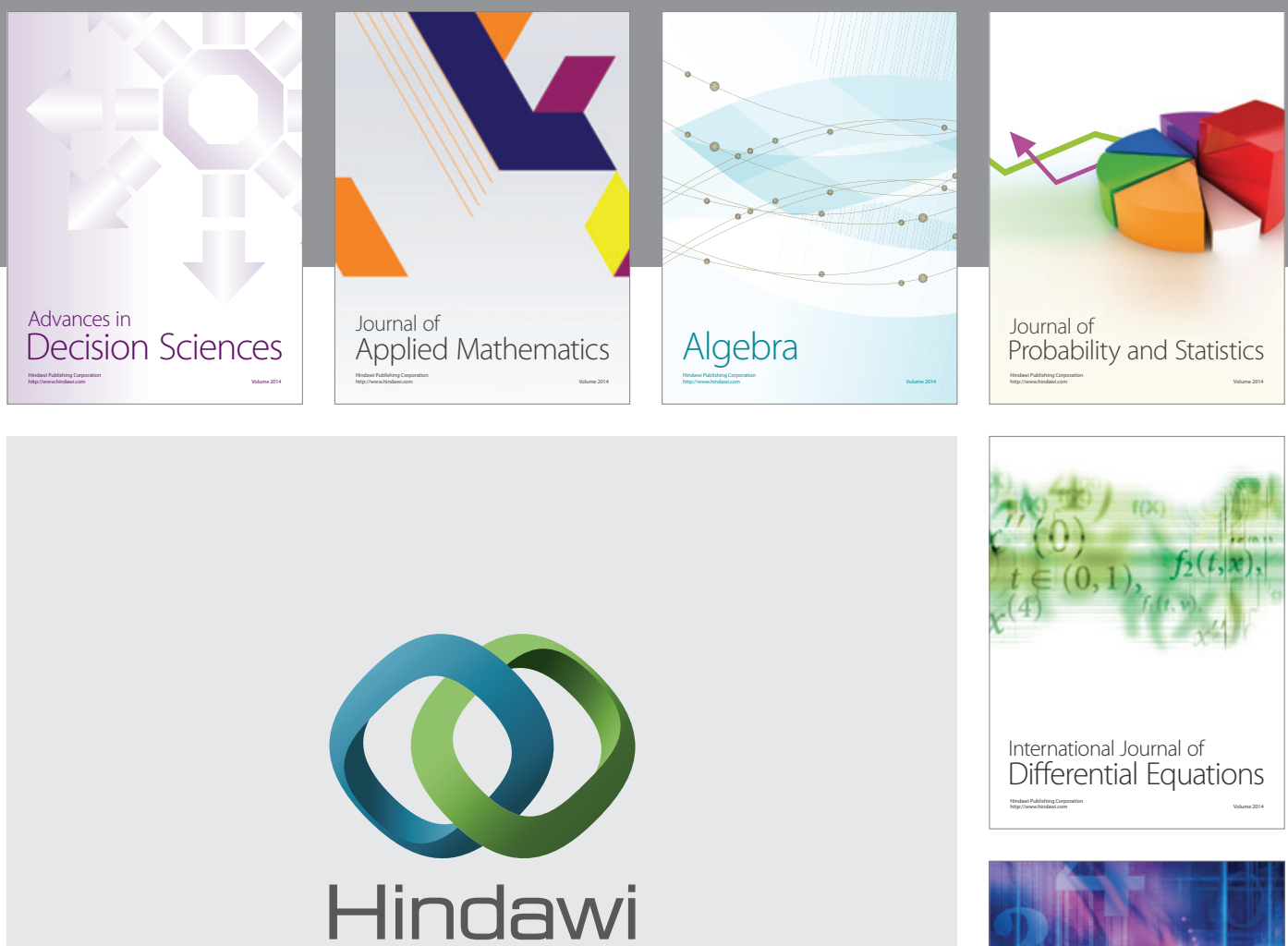

Submit your manuscripts at http://www.hindawi.com
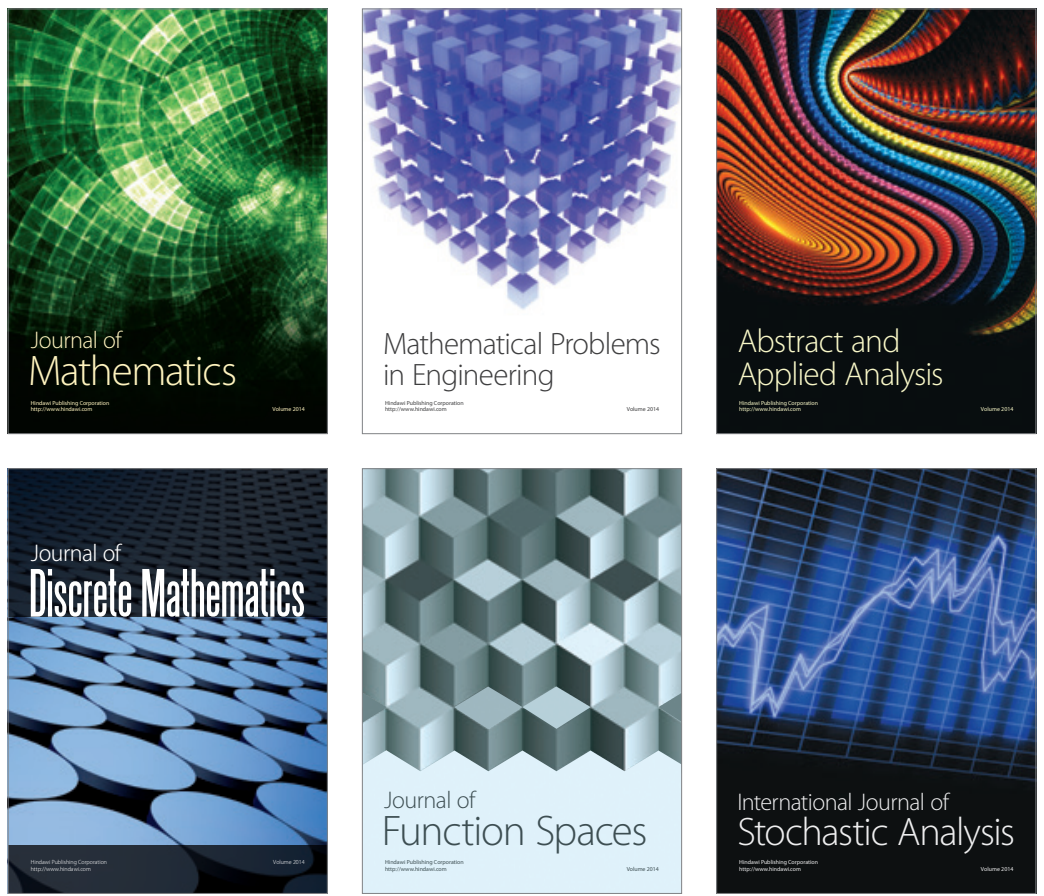

Journal of

Function Spaces

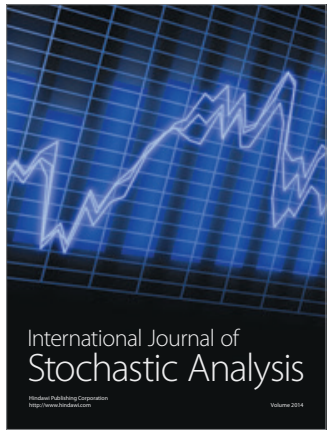

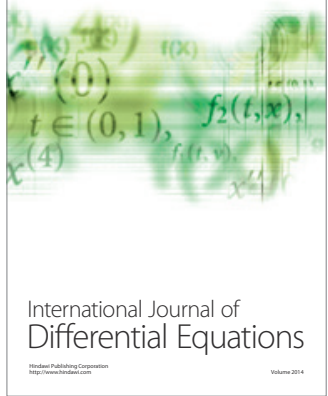
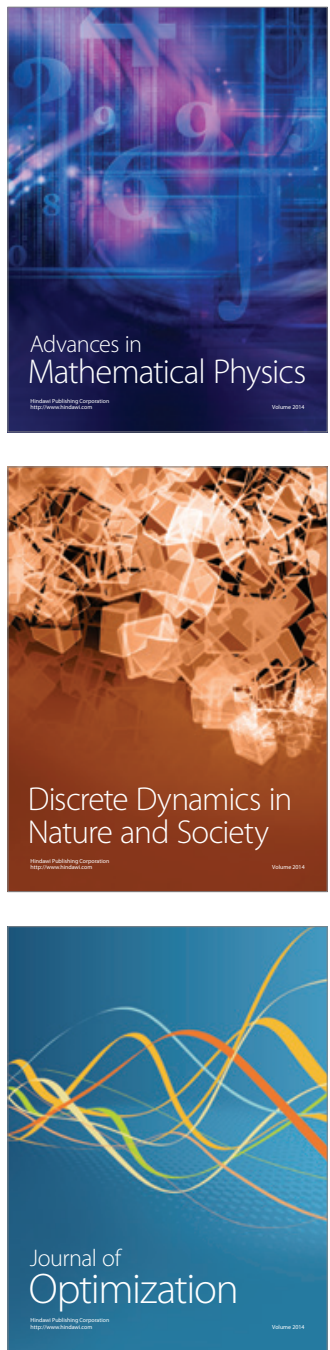